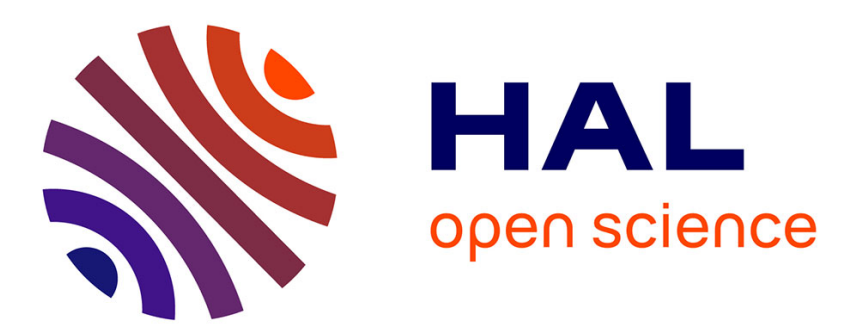

\title{
Ueber die experimentelle Syphilis
}

Elie Metchnikoff, Emile Roux

\section{To cite this version:}

Elie Metchnikoff, Emile Roux. Ueber die experimentelle Syphilis. Deutsche medizinische Wochenschrift, 1903, 29 (50), pp.943-944. pasteur-00557981

\section{HAL Id: pasteur-00557981 https://hal-pasteur.archives-ouvertes.fr/pasteur-00557981}

Submitted on 20 Jan 2011

HAL is a multi-disciplinary open access archive for the deposit and dissemination of scientific research documents, whether they are published or not. The documents may come from teaching and research institutions in France or abroad, or from public or private research centers.
L'archive ouverte pluridisciplinaire HAL, est destinée au dépôt et à la diffusion de documents scientifiques de niveau recherche, publiés ou non, émanant des établissements d'enseignement et de recherche français ou étrangers, des laboratoires publics ou privés. 
zu sein in dem Kampfe gegen die furchtbarsten Feinde der Menschen und Thiere, in dem Kampfe gegen die krankheitserzeugenden Mikroorganismen, welchem er sein ganzes, arbeitsreiches Leben geweiht!

\section{Aus dem Institut Pasteur in Paris. Ueber die experimentelle Syphilis.}

\section{Von EI. Metschnikoff und Em. Roux.}

Die anthropoïden Affen sind bereits einige Male zu Versuchen über rein menschliche Krankheiten verwendet worden. So hat R. Koch Orang-Utans mit dem Virus von Malariafieber geimpft. Nur erwiesen sich diese Thiere als vollständig immun gegenüber Malaria, welche ausscliliesslich für den menschlichen Organismus pathogen zu sein scheint. Grünbaum in Liverpool kam zu positiven Ergebnissen in seinen Versuchen, den Abdominaltyphus durch Infektion per os bei Schimpansen zu erzeugen.

Wir selbst haben einem jungen Schimpansen etwas Melanosarkomgewebe in die vordere Augenkammer und unter die Haut eingeimpft. Dieser Versuch hat uns indessen zu keinem sicheren Resultate geführt. Daher haben wir uns entschlossen, einem anderen von uns angeschafften, jungen, weiblichen Schimpansen etwas syphilitisches Virus einzuimpfen. Diesmal war das Ergebniss durchaus positiv, sodass wir in den Stand gesetzt wurden, in einer Sitzung der Pariser medizinischen Akademie einen experimentell erzeugten harten und durchaus charakteristischen Schanker zu demonstriren. Die anwesenden Mitglieder und darunter Spezialisten, wie die Herren Fournier, du Castel und Hallopeau, erklärten sich einstimmig für die Syphilisnatur der ihnen gezeigten Läsion. Die lange Inkubationsdauer (25 Tage), die sämmtlichen Merkmale, sowie die ganze Art der Entwickelung zeigten in unzweideutiger Weise, dass es sich wirklich um eine primäre syphilitische Erscheinung,handelte, zumal der Schanker von einer ganzen Reihe fast gleichzeitig entwickelter Lymphdrüsen an der entsprechenden Seite der Leistengegend begleitet war.

Einen Monat nach dem Erscheinen des harten Schankers entwickelten sich Hautpapeln auf verschiedenen Theilen der Körperoberfläche. Es haben sich im ganzen etwa 15 solcher squamösen Papeln ausgebildet, wovon einige auf den Schenkeln, andere auf dem Bauche resp. auf dem Rücken erschienen. Rund und von der Grösse eines Zwanzigpfennigstückes liessen diese Papeln eine peripherische Zone unterscheiden, welche anfänglich roth war, später stark pigmentirt wurde; die mittlere Zone erschien schmal und weisslich und begrenzte eine grosse centrale Schuppe. Das Ganze zeigte eine trockene Oberfläche; es genügte indessen, die Papeln etwas zu kratzen, um eine trübe Flüssigkeit zu erhalten.

Die Papeln haben sich in gleichem Zustande einen ganzen Monat erhalten, worauf sie sich rückzubilden begannen. Sie blieben jedoch bis zum Tode des Thieres bestehen, welcher ungefähr 14 Wochen nach der Verimpfung des syphilitischen Virus erfolgte. Der Tod ist durch eine verallgemeinerte Pneumococcie verursacht worden, deren Ausgangspunkt höchst wahrscheinlich in einer Gingivitis lag. Es ist schwer, sich über die Natur dieser letzteren Affektion auszusprechen, zumal da dieselbe sich sehr oft bei in Gefangenschaft gehaltenen Schimpansen entwickelt. Somit ist es unmöglich, an ihrem syphilitischen Ursprung festzuhalten.

Unser erster Versuch zeigte uns also die Möglichkeit, eine experimentelle Krankheit bei einem anthropoïden Affen zu erzeugen, welche durchaus der menschlichen Syphilis ähnlich ist. Es handelt sich um eine Krankheit, welche sich zunächst auf dem Impfungsorte lokalisirt, welche dann die lymphatischen Organe ergreift und zur Entwickelung eines Hautausschlages führt.

Dieses erste Resultat schien uns zur Genüge ermuthigend, um weitere Versuche vorzunehmen. Es müsste zunächst festgestellt werden, dass die experimentelle Syphilis des ersten Schimpansen kein Zufall war und dass sie einer weiteren Fortpflanzung fähig ist. Unsere erste Aufgabe war somit, zu bestimmen, ob das syphilitische Virus des ersten Affen auf ein anderes Individuum derselben Gattung erfolgreich überimpft werden kann. Zu diesem Zwecke haben wir syphilitische Produkte des von uns beschriebenen Falles auf einen männlichen, etwa fünf Jahre alten Schimpansen (zur Varietät Trogdodytes calvus gehörig) fortgepflanzt. Wir verdanken dieses Thier der Liebenswürdigkeit des Herrn Gazengel, Grundbesitzer in der französischen Kongokolonie, sowie der Zuvorkommenheit der Verwaltung des Zoologischen Gartens in Paris, namentlich des Herrn Prof. Oustalet. Wir sprechen diesen Herren unseren öffentlichen Dank für ihre Freundlichkeit aus.

45 Tage nach dem Erscheinen des harten Schankers beim ersten Schimpansen, d. h. zu einer Zeit, wo diese syphilitische. Erscheinung bereits auf dem Wege der Heilung war, haben wir von ihr etwas Flüssigkeit genommen und auf den Penis unseres neuen Thieres mit Hülfe eines Skarifikators verimpft. Da es indessen möglich gewesen wäre, dass ein so altes Produkt bereits seine Virulenz verloren hätte, so haben wir zu gleicher Zeit auf den linken Oberschenkel unseres Männchens etwas Flüssigkeit aus einer squamösen Papel des syphilitischen Affen verimpft.

Die ersten Tage nach diesen beiden Impfungen verliefen ohne irgend welche Krankheitserscheinung. Die ganz oberflächlichen Läsionen, welche mit dem Skarifikator von Vidal erzeugt wurden, heilten sofort, ohne Spuren zu hinterlassen. Erst acht Tage nach der Inokulation zeigten sich auf dem Oberschenkel zwei kleine, vorübergehende Geschwüre. Mehrere Tage lang erschien bei unserem Thiere kein krankhaftes Symptom, welches man auf Syphilis beziehen konnte, zumal die zwei eben erwähnten Geschwüre sich weder hart anfühlten, noch von einer Lymphdrüsenschwellung begleitet waren. Uebrigens dauerten diese kleinen Geschwüre nur wenige Tage.

Erst am 35. Tage nach der Impfung konnten wir auf der linken Seite des Penis eine kleine oberflächliche Erosion feststellen. Sie war breit wie eine kleine Linse und erschien weder geröthet noch hart. An den folgenden Tagen wurde sie grösser, speziell in die Länge gezogen. Zu gleicher Zeit entwickelte sich auf dem geimpften Oberschenkel eine zweite Erosion, von einem leicht indurirten Gewebe umgeben. Die Lymphdrüsen der Inguinalgegend waren auf beiden Seiten zu fühlen.

An den folgenden Tagen machte das Geschwür am Penis grosse Fortschritte. Die Läsion des Oberschenkels zeigte zu gleicher Zeit eine geschwürige centrale Partie; sie war geröthet und von einem deutlichen Wall umgeben. Nach der Ansicht des Herrn Prof. Fournier, welcher die Güte hatte, unseren Affen zu besichtigen, "machte die Gestalt dieser Läsion ganz den Eindruck eines oberflächlichen Schankers der Haut." Die Lymphdrüsen haben sich inzwischen auf der den Geschwüren des Penis und des Oberschenkels entsprechenden Seite vergrössert. Man konnte sehr deutlich zwei unter der Haut bewegliche, schmerzlose, harte Lymphdrüsen wahrnehmen. Die eine war von der Grösse einer grossen Erbse, die andere erreichte die Grösse eines Kirschkernes.

Einen Monat nach seinem Erscheinen begann der Schanker des Oberschenkels zu heilen. Derjenige des Penis dagegen entwickelte sich weiter. Die Lymphdrüsen der linken Inguinalgegend wiesen eine ansehnliche Volumvergrösserung auf.

In der Folge heilte der Schanker des Oberschenkels weiter; auch die Lymphdrüsen zeigten eine Grössenabnahme, während der Schanker des Penis sich in demselben hochentwickelten Zustande bis zum Tode des Thieres erhielt, welcher 45 Tage nach dem Erscheinen der ersten syphilitischen Symptome erfolgte.

Die letzten 14 Tage seines Lebens litt der Schimpanse an Schnupfen und hustete oft. Der Appetit wurde immer geringer, während die allgemeine Schwäche allmählich zunahm.

Das Thier starb, ohne irgend welche sekundäre Syphilissymptome aufgewiesen zu haben. Bei der Sektion konnte man auch keine auf Syphilis zu beziehende Erscheinung wahrnehmen. Die fettige Entartung der Nieren und der Leber, sowie einige geringe Geschwürsbildungen der Verdauungsorgane müssen eher durch allgemeine Infektion mit einem kleinen, influenzaähnlichen Bacillus erklärt werden. Dieser Bacillus entwickelte sich in grosser Menge aus dem auf Agar überimpften Herzblute.

Der Schimpanse, dessen Krankheitsgeschichte wir geschildert haben, hat uns einen weiteren Beweis dafür geliefert, dass diese Anthropoïdengattung für das Syphilisvirus sehr empfindlich ist. Dieser Versuch hat uns ferner gezeigt, dass die primären Syphiliserscheinungen des Schimpansen die grösste Aehnlichkeit mit denjenigen des Menschen aufweisen. Schliesslich bewies er, dass das syphilitische Virus von einem Schimpansen auf den anderen überpflanzt werden kann. Auf Grund dieser Ergebnisse kommen wir zu der Schlussfolgerung, dass das Studium der mensch- 
lichen Syphilis durch Versuche an menschenähnlichen Affen sehr gefördert werden kann.

\section{Der Antheil Koch's an der Malaria- forschung.}

Von Major Ronald Ross, F. R. S., O. B., Professor der Tropenmedizin an der Universität Liverpool.

Mit ganz besonderer Freude entspreche ich dem ehrenvollen Auftrag der Redaktion, einen Artikel zu dieser Nummer beizusteuern, und ergreife die Gelegenheit, einen kurzen Ueberblick über die Arbeiten zu geben, welche Geheimrath Prof. Koch auf dem wichtigen Gebiete der Malaria geleistet hat. Viele Arbeiten freilich sind gerade in der neuesten Zeit über Malaria geschrieben worden, manche von Schriftstellern, welche, wie ich fürchte, nur sehr wenig selbständige Kenntniss der Materie und sogar der einschlägigen Litteratur besassen; andere wiederum von solchen, die zwar mit der Feder Entdeckungen zu machen suchten, die aber durch experimentelle Untersuchungen ihre Widerlegung fanden. Als Folge hiervon hat eine unbegrenzte Verwirrung in die Geschichte dieses Zweiges unserer Wissenschaft sich eingeschlichen, und sind die tüchtigsten und zuverlässigsten Arbeiten nahe daran, gegenüber den inhaltslosesten und unzuverlässigsten in Vergessenheit zu gerathen. Unter die ersteren - unter die fundamentalen Untersuchungen der Malaria - müssen wir diejenigen von Koch und seinen tüchtigen Mitarbeitern einreihen; und alle diejenigen, welche auf dem gleichen Gebiete getreulich mitgearbeitet haben, werden stets sich vereinen, um Jenen die gebührende Ehre zu erweisen.

Man wird sich erinnern, dass Laveran im Jahre 1880 die Hämatozoën der Malaria entdeckte. Drei Jahre später kamen King und Koch vollkommen gleichzeitig auf die Vermuthung, dass die Infektionskrankheit durch die Stiche von Mosquitos herbeigeführt sein könnte. King's Anschauungen basirten auf der älteren Litteratur, die jetzt allem Anschein nach keine Berücksichtigung mehr findet. Er kannte nicht Laveran's Beobachtung, doch waren ihm Manson's Untersuchungen bekannt, welche bewiesen, dass die Embryonen von Filaria nocturna sich in Mücken entwickeln; und er übergab seine Hypothese der Oeffentlichkeit in einer mit grosser Gewandtheit und Genauigkeit verfassten, vorzüglichen Schrift im Jahre 1883. Auf der anderen Seite waren Koch's Vermuthungen, die er während seiner indischen Expedition im Jahre 1883-84 gehegt hatte, nur in seinen mündlichen Vorträgen öffentlich bekannt geworden; - freilich bezog sich R. Pfeiffer auf sie später in einer gedruckten Abhandlung. Laveran äusserte die gleiche Ansicht nur in einem kurzen, mit einem Fragezeichen versehenen Satze aus dem Jahre 1884; und 10 Jahre später führte sie Manson, gestützt auf neue Gründe, weiter aus, nämlich auf die Thatsache, dass die sogenannte Flagellaform aus den Capillaren des Wirthes ausschlüpft. Wenige Historiker erweisen diesen interessanten Vermuthungen genügend Gerechtigkeit, von denen jede unabhängig von der andern ausgesprochen wurde; indessen ist doch woh die Hypothese, die so oft die Forschung erst einleitet, kaum weniger wichtig, als die letztere selbst. Wir müssen sicher Koch das Verdienst zusprechen, dass er einer der ersten gewesen ist, welcher mit Bezug auf die Beantwortung der enorm wichtigen Frage über den Infektionsweg der Malaria das Richtige vorausgesehen hat.

Aber Koch begnügte sich nicht mit einer Hypothese, und unter allen den Genannten, welche nur theoretisch sich geäussert hatten, war er der einzige, der nach einer experimentellen Bestätigung selbständig suchte. Auf seiner Expedition nach Afrika im Jahre 1897 sammelte er viele werthvolle Thatsachen zu Gunsten der Mosquito-Hypothese - unter anderen Beobachtungen, zu denen die Feststellung der Fieberkurve der Tropicaform und ihre Beziehungen zum Entwickelungsverlauf ihrer Parasiten (der Halbmondbildner) gehört - , und er wies auch auf die Analogie mit dem Texasfieber der Rinder hin, welches durch Zecken hervorgerufen wird. Seine Gesichtspunkte, für welche er mit Festigkeit eintrat, waren unabhängig von denen Manson's und von meinen eigenen aufgestellt, und waren thatsächlich im Juni 1898 veröffentlicht worden; neun Tage, bevor Manson meine Erfolge bei der Züchtung von Proteosoma in den Mosquitos der Gattung Culex ankündigte. Gleichzeitig hatte Koch sich mit der Färbung der sogenannten Flagellaform beschäftigt, und hatte in Uebereinstimmung mit einer von Bignami und Bastianelli ausgesprochenen Annahme (Lancet 1898, Vol. II, S. 1620) einen weiteren Erfolg dadurch gehabt, dass er das Chromatin in ihnen darstellte, während er im Hospital Santo Spirito in Rom im Sommer 1898 arbeitete. Diese Beobachtung erschütterte endgültig die Theorie der Italiener vom "Agonezustande" und belehrte sie zweifellos auch über die nöthige Technik, - denn wir finden, dass Bignami und Bastianelli die gleiche Thatsache unmittelbar nachher entdeckten (siehe dieselbe Nummer des Blattes). Koch war nun im Stande, mit Kossel die wahre Natur der Flagella festzustellen und zu zeigen, dass sie für die Befruchtung der Makrogameten und für die Erzeugung der "Würmchen“ bestimmt sei. Die Thatsache, dass er, ohne es damals zu wisssen, in diesen Untersuchungen durch Sacharoff und Mc Callum überholt worden war, thut dem Verdienste seiner Leistungen keinen Eintrag. Unabhängig hat er auf "jenen Hauptpunkt in der Malaria-Theorie hingewiesen, welchem der Befund des wechselnden Wirthes zu Grunde liegt.

Indessen bestand gerade hier die grösste Schwierigkeit; wir standen zwei unbekannten Faktoren gegenüber - nämlich einerseits hinsichtlich der Gattung von Mosquitos, die in Betracht kam, andererseits hinsichtlich der Gestalt und Lagerung der Parasiten in ihnen. Der eine Faktor konnte nicht mit Sicherheit festgestellt werden, ohne dass der andere gefunden war; die Entdeckung des einen schloss diejenige beider in sich. Man besass keine deutlichen Anzeichen, die dafür verwerthet werden konnten, die krankheitsübertragende Gattung Mosquito ausfindig zu machen. Es gab keine Möglichkeit, die unschädlichen Arten abzusondern und zwar gerade in den von der Malaria besonders ergriffenen Ortschaften; und wir wissen heute sogar, dass die schädliche Spezies auch dort in reichlicher Menge vorkommt, wo man von Malaria noch niemals gehört hat. Das Problem konnte daher lediglich durch eine Methode gelöst werden, zu welcher wir so oft im äussersten Falle gedrängt werden - - durch die Methode des Probirens. Mosquito nach Mosquito wurde untersucht, freilich nur mit der sehr geringen Wahrscheinlichkeit, dabei Erfolg zu haben; und es kann wohl kaum Wunder nehmen, dass Koch nicht seine ganze Zeit einer so langen und vielleicht fruchtlosen Untersuchung widmen konnte, was nun aber doch nöthig gewesen wäre. So fiel denn diese Arbeit auf mich: Vom April 1895 an versuchte ich mehr als zwei Jahre lang die Parasiten in meinen "grauen" und "rostbraunen" Mosquitos (Culex und Stegomyia) zu züchten, ohne jeden Erfolg; jedoch im August und September 1897 fand ich schliesslich in drei Individuen, die zu zwei Spezies einer neuen Gattung Mosquitos gehörten (nämlich zu meinen mit gefleckten Flügeln versehenen Mosquitos oder Anopheles), etwas, was, wie ich sofort sah, Entwickelungsstufen der Parasiten war. Wie vorausgesetzt, wurde gleichzeitig hierdurch sowohl die Gattung Mosquito, die in Betracht kam, als auch Form und Lagerung der Parasiten in ihr aufgefunden. Behindert durch die Theilnahmslosigkeit der maassgebenden Autoren im Augenblick meines Erfolges, wurde ich gezwungen meine Arbeit nur insoweit fortzusetzen, als ich Proteosoma der Vögel studierte; schliesslich konnte ich den Entwickelungsgang dieser Parasiten dadurch vollständig verfolgen, dass ich eine grössere Anzahl von Sperlingen durch die Bisse von Mosquitos infizirte; dies geschah im Juli 1898. Nun musste ich wiederum pausiren, denn, obwohl ich sowohl die Regierung von Indien wie die Royal Society um Hilfe anging, wurde ich genöthigt Indien zu verlassen und war nicht fähig die Erforschung der menschlichen Malaria durchzuführen, bis im August 1899 durch die Hilfe von Annett und Austen meine Untersuchungen erfolgreich durchgeführt wurden, und zwar in wenigen Wochen in Sierra Leone. ${ }^{1}$ )

Manson berichtete über meinen Erfolg mit Proteosoma in dem Augenblicke, als Koch die „Würmchen" in Italien aufgefunden hatte, also im Sommer 1898 . Koch und R. Pfeiffer bestätigten sofort meine Arbeit, indem sie jene Parasiten in Culex nemorosus züchteten; das geschah in Rom im September, und Mitte November konnten sie in Berlin den Entwickelungscyklus bis zu Ende durchführen. Ihre Veröffentlichung wurde zwar durch die Herstellung zahlreicher beweisender Photogramme lange hingezogen; dennoch gebührt ihnen sicher das Verdienst der

1) Diese Bemerkungen sind nothwendig, um Koch's Stellung mit Bezug auf die einzelnen Ereignisse klar zu stellen. 